DOI: $10.30612 /$ re-ufgd.v4i8.7791

\title{
PRODUÇÃO DE FENO ORGÂNICO COMO ESTRATÉGIA DE SUPLEMENTAÇÃO VOLUMOSA PARA RUMINANTES PRODUZIDOS NAS COMUNIDADES RURAIS DE MATO GROSSO DO SUL
}

Organic hay production as roughage supplementation strategy for ruminants produced in rural communities of Mato Grosso do Sul

\author{
${ }^{1}$ Euclides Reuter de Oliveira; ${ }^{1}$ \\ ${ }^{2}$ Elaine Barbosa Muniz; \\ ${ }^{1}$ Andrea Maria de Araújo Gabriel; \\ ${ }^{3}$ Flávio Pinto Monção; \\ ${ }^{1}$ Jefferson Rodrigues Gandra; \\ ${ }^{1}$ Érika Rosendo de Sena Gandra; \\ ${ }^{4}$ Thaís Lemos Pereira; \\ ${ }^{1}$ Mábio Silvan José da Silva; \\ ${ }^{5}$ Willian da Silva Gouvea; \\ ${ }^{5}$ Adrielly Aparecida do Carmo; \\ ${ }^{5}$ Cibeli de Almeida Pedrini; \\ ${ }^{5}$ Roni Ailson Stefanes Becker
}

\section{Recebido em 26/03/2017 Aceito em 15/12/2017}

Resumo: Objetivou-se por meio deste evento relacionar aspectos referentes à implantação de uma área demonstrativa na UFGD para a produção de feno orgânico, visando proporcionar suporte na alimentação de bovinos de corte nas comunidades rurais de Dourados/MS. Assim, foram efetuados enfoques caracterizados como: forrageiras indicadas para fenação; etapas do processo de fenação; qualidade e valor nutritivo do feno; perdas durante o processo de fenação; uso do feno pelos animais e avaliação econômica da utilização do feno. $\mathrm{Na}$ prática, houve demonstração da implantação, corte, desidratação da forragem verde, enleiramento, viragem, enfardamento e armazenamento. No processo de enfardamento utilizou-se uma enfardadeira horizontal acoplada ao trator e foram realizadas análises laboratoriais para servir como banco de dados aos produtores e aos demais interessados. O evento foi divulgado por meio do contato verbal, cartazes e por distribuição de folders em locais estratégicos. O evento proporcionou uma demonstração teóricoprática dos processos de fenação, com participação de produtores rurais, assentados, quilombolas e discentes de vários cursos. O evento supriu as necessidades de conhecimento do público, além de oferecer conhecimento de manejo vegetal de forma orgânica.

Palavras-Chave: Extensão. Fenação. Forragem. Produção animal.

\footnotetext{
${ }^{I}$ Universidade Federal da Grande Dourados, Dourados, MS, euclidesoliveira@ufgd.edu.br; ${ }^{2}$ UNIOESTE, Marechal Cândido Rondon, PR, ebmuniz@yahoo.com.br; ${ }^{3}$ Pós Doutorando da Unimontes/Janaúba-MG, moncaomoncao@yahoo.com.br; ${ }^{4}$ Mestranda de Zootecnia da UFGD, thais-lemos01@hotmail.com; ${ }^{5}$ Discentes do curso de Zootecnia da UFGD.
} 
Abstract: The objective of this event was to describe all aspects related to the implementation of a demonstration area, at the Federal University of Grande Dourados (UFGD), for organic hay production aiming to provide support in the feeding of beef cattle in the rural communities of Dourados/MS. Therefore, the hay day field demonstration featured information about the forage species for hay production, stages of the haymaking, quality and nutritional value of hay, losses during the haymaking process, the use of hay in animal feeding and economic evaluation of hay utilization. In practice, there was a demonstration of the steps of haymaking: implantation, mowing, tedding of the green forage, raking, baling and storage. A horizontal baler was used to bale the hay, and it was coupled to the tractor. The laboratory analysis was performed to serve as a database to producers and other interested parties. The event was publicized through verbal contact, posters, and distribution of folders at strategic locations. The field day provided a theoretical-practical demonstration of the processes of haymaking, with the participation of rural producers, settlers, quilombolas and students from several courses. Overall, the event meet the knowledge needs of the public, as well as providing knowledge about organic management.

Key words: Extension. Haymaking. Forage. Animal Production.

\section{Introdução}

A produção de ruminantes no Brasil baseia-se no uso de plantas forrageiras como principal fonte de nutrientes para os animais ao longo do ano (BARBERO et al., 2015). De acordo com os mesmos autores, isso ocorre porque nesses sistemas, o custo de produção é, normalmente, inferior aos sistemas intensivos de produção, tornando o produtor brasileiro cada vez mais competitivo no mercado interno e externo.

No entanto, a produção de forragem ao longo do ano não é constante, afetando a produção animal. Dessa forma, estratégias de suplementação dos animais são necessárias para continuidade do desempenho animal e a mantença do produtor rural no campo. Dentre as estratégias de suplementação, a produção de feno é uma técnica que existe desde tempos remotos. Mesmo assim, os produtores atuais, principalmente os pequenos produtores, ainda são muito dependentes de informações básicas que varia desde a escolha da melhor forrageira a ser fenada ao processo de fenação propriamente dita. Isso acontece porque os pequenos produtores nem sempre tem disponibilidade de recursos para contratar técnicos para orientação ou tempo para participar de eventos privados com tal finalidade.

Sendo assim, a extensão rural nas Universidades aliada com as informações geradas pela pesquisa tem o grande desafio de acessar os produtores e transmitir as informações necessárias e acompanhar sua aplicação no desenvolvimento rural (PEIXOTO, 2008; GABRIEL et al., 2015).

A extensão rural se caracteriza de várias formas, sendo destaque para formação profissional do cidadão e se credencia, cada 
vez mais, junto à sociedade como espaço privilegiado de produção do conhecimento significativo para a superação das desigualdades sociais existentes, como prática acadêmica que interliga a Universidade nas suas atividades de ensino e de pesquisa, com as demandas da maioria da população (SHEIDEMANTEL et al.,2004; OLIVEIRA et al., 2012).

Os conhecimentos gerados nas Universidades muitas vezes não chegam às pessoas que necessitam destas informações. Diante disto, a extensão tem um papel fundamental para a democratização do acesso a essas informações que irá promover a aplicação dos resultados de tais pesquisas na prática e observando os seus resultados alcançados (OLIVEIRA et al., 2012; 2016d).

Uma das formas de promover esta aliança entre pesquisa e extensão é através do dia de campo que é um método de difusão de tecnologias agropecuárias no meio rural eficiente e de baixo custo. Este é um método antigo utilizado no Brasil que vem sendo usado desde a implantação do Serviço de Extensão Rural. O dia de campo poderá ser desenvolvido para diferentes objetivos tais como exploração de culturas, criações e agroindústrias entre outros. Podendo tratar ao mesmo tempo, de um ou vários temas referentes a uma ou mais explorações agropecuárias e/ou agroindustriais (SILVA e
SILVA, 2013). O dia de campo, independentemente de ser de dia ou à tarde, busca despertar o interesse e a aplicação mais rápida da tecnologia que está sendo apresentada. As pessoas vão adquirindo o conhecimento e descobrindo qual o melhor caminho a seguir em função das suas necessidades no meio rural (SILVA e SILVA, 2013).

Atualmente, uma necessidade que vem crescendo no campo é a produção de alimentos orgânicos. Existem cerca de dois milhões de agricultores orgânicos estabelecidos em $80 \%$ dos países em desenvolvimento, movimentando 60 bilhões de dólares em produtos anualmente. A ascensão do mercado de produtos naturais e orgânicos segue uma tendência mundial de aumento da demanda por produtos e serviços que proporcionam saúde e bem-estar (FEIDEN et al., 2002). Soma-se a esse fator a crescente desconfiança de alguns setores da sociedade em relação à indústria moderna, que trouxe uma série de facilidades à vida cotidiana. Aumentou, também, significativamente a manipulação de químicos persistentes no meio ambiente, com graves consequências para a saúde humana e para os ecossistemas naturais (DIAS et al., 2015).

Com base no exposto, objetivou-se a avaliar a importância do dia de campo com 
ênfase na demonstração das técnicas envolvidas na produção de feno orgânico para produtores rurais, alunos e técnicos envolvidos com a agropecuária da região da Grande Dourados e Centro-Sul do Mato Grosso do Sul.

\section{Materiais e Métodos}

A ação de extensão, Produção de feno orgânico, vem sendo desenvolvido na Faculdade de Ciências Agrárias da Universidade Federal da Grande Dourados desde o ano de 2007. Mas foi abordada a ação ocorrida no ano de 2016, que apresentou carga horária total de 500 horas. Sendo de abrangência regional, onde o público alvo foram os produtores rurais, estudantes universitários, professores, técnicos agrícolas e a comunidade geral da Grande Dourados.

Vários estudos, debates, congressos foram realizados sobre os problemas da alimentação animal, visando aprimorar os métodos ou procedimentos até então adotados para aumentar a oferta de carne e leite.

Em novembro de 2016, foi realizado um dia de campo nas dependências da Faculdade de Ciências Agrárias/UFGD, Dourados, MS abordando temas atuais relacionados à produção animal/vegetal com ênfase na produção de feno no processo orgânico. Os enfoques do trabalho foram: forrageiras indicadas para o processo de fenação, etapas do processo de fenação, qualidade e valor nutritivo do feno, perdas durante o processo de fenação, uso do feno pelos animais, avaliação econômica da utilização do feno e as formas de adubação orgânica conforme o Ministério da Agricultura.

$\mathrm{Na}$ prática, foi realizada a demonstração da implantação, corte, desidratação da forragem verde, enleiramento, viragem, enfardamento e armazenamento. No processo de enfardamento foi utilizada uma enfardadeira horizontal acoplada ao trator, e amostras foram coletadas para realizar análises laboratoriais para servir como banco de dados aos produtores e aos demais interessados.

Acoplado ao dia de campo, experimentos prévios foram realizados comparando genótipos de Cynodon com potencial promissor para produção de forragem na região. Além disso, foi avaliada a melhor idade de corte de cada genótipo (OLIVEIRA et al., 2013; 2014; 2016abce; MONÇÃO et al., 2014; 2016).

O evento foi divulgado por meio de contato verbal, cartazes e por distribuição de folders em locais estratégicos.

\section{Resultado e Discussão}


No campo agrostológico da UFGD/FCA existe o cultivo de várias espécies e cultivares de forrageiras com potencial de utilização para produção de feno. Esse contato do público com as plantas foi fundamental na geração de novos conhecimentos. Na prática, foi possível abordar os pontos positivos e negativos envolvidas nas escolhas corretas dos genótipos para fenação. Além disso, o contato dos produtores com genótipos com outras finalidades, como os cultivares do gênero Pennisetum, despertou outras possibilidades de suplementação dos animais.

No que tange a fenação, o enfoque foi para os genótipos de Cynodon. Práticas de curtição do esterco e de outras fontes de matéria orgânica foi abordada e demonstrada no evento, o que possibilitou a geração de conhecimentos sólidos por parte dos integrantes sobre as práticas orgânicas utilizadas nesse sistema de produção. A adoção do sistema orgânico vai de encontro com o desenvolvimento sustentável que estabelece que as necessidades da atual geração sejam atendidas sem comprometeras gerações futuras, ou seja, não ocorrendo o esgotamento dos recursos naturais. Trabalhar de forma sustentável envolve estudar, planejar e programar ações sempre pensando no hoje e no amanhã, abordando os aspectos econômicos, sociais e ambientais (MAZZOLENI e NOGUEIRA, 2006).

Houve esclarecimento entre os principais cuidados nos procedimentos de execução de enfardar para os produtores rurais, estudantes profissionais da área. $\mathrm{O}$ enfardamento não é condição única para a produção de feno, mas é condição prática que facilita 0 armazenamento, transporte, manuseio e comercialização. O objetivo é reduzir o volume de forragem pela compactação, aumentado à densidade. Isso irá proporcionar maior quantidade de feno que poderá ser armazenada em menor espaço. Quando se armazena em fardos, o feno tem melhor conservação e também possibilita um maior controle da quantidade disponível (EVANGELISTA e LIMA, 2013).

O trabalho gerou dados com as análises laboratoriais realizadas na UFGD e esses foram colocados em um banco de dados que futuramente poderá ser utilizado para uma consulta sempre que for necessário. Os bancos de dados são importantes para que se possa, em algum momento, serem utilizados em pesquisas, para efeitos comparativos com outros trabalhos e outras situações que julgar necessário. Na Tabela 1, pode ser verificado os resultados da composição químico bromatológicas (DETMANN et al., 2012) dos diferentes genótipos na idade de corte de 50 dias. 
Tabela 1. Valores médios da composição bromatológica dos diferentes genótipos pertencente ao gênero Cynodon.

\begin{tabular}{lcccccc}
\hline \multirow{2}{*}{ Nutrientes } & \multicolumn{7}{c}{ Feno } \\
\cline { 2 - 7 } & Jiggs & Vaquero & Tifton 68 & Coast- & Tifton 85 & Russel \\
& & & & & \\
Cross & \\
\hline MS & 92,65 & 92,86 & 92,56 & 92,88 & 92,43 & 92,58 \\
PB $^{1}$ & 12,63 & 10,98 & 12,3 & 10,36 & 11,91 & 11,47 \\
FDN $^{1}$ & 55,23 & 55,91 & 54,68 & 57,98 & 56,09 & 56,20 \\
FDA $^{1}$ & 23,68 & 21,60 & 24,15 & 25,03 & 23,69 & 23,94 \\
Lig $^{1}$ & 3,97 & 4,08 & 4,37 & 4,65 & 4,34 & 4,43 \\
NDT $^{1}$ & 70,46 & 72,08 & 70,09 & 69,40 & 70,46 & 70,26 \\
MM $^{1}$ & 6,36 & 6,07 & 6,21 & 5,92 & 5,78 & 6,12 \\
\hline
\end{tabular}

${ }^{1}$ Base da MS; NDT estimado de acordo com Patterson et al. (2000); MS: matéria seca; PB: proteína bruta; FDN: fibra em detergente neutro; FDA: fibra em detergente ácido; Lig: lignina; NDT: nutrientes digestíveis totais; MM: matéria mineral (Fonte: OLIVEIRA et al., 2014ab).

A partir dos resultados da pesquisa foi possível apresentar ao público a melhor idade de corte tendo a produção de matéria seca e teor de proteína bruta como variáveis impactantes no sistema de produção animal. A produção de matéria seca envolve a eficiência e a diluição dos custos fixos de produção e o teor de proteína bruta, como nutricional mais oneroso da dieta dos animais. Sendo assim, foi estabelecida para a região uma melhor idade de corte (50 dias quando o genótipo é manejado no outonoinverno) dos genótipos avaliados (Figura 1). 


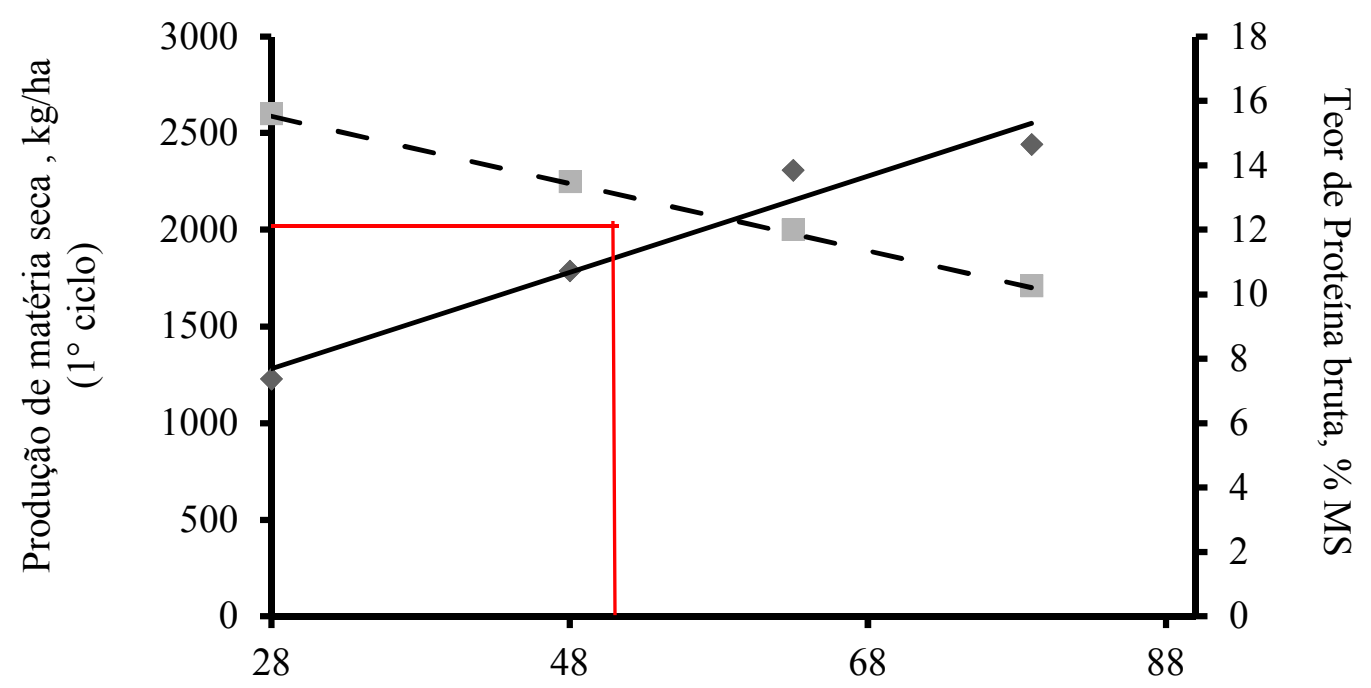

- Produção de Matéria seca

Proteína bruta

Figura 1. Produção de matéria seca e teor de proteína bruta de diferentes genótipos de Cynodon em diferentes idades de corte.

Essa transferência de tecnologias entre o meio acadêmico e o campo é o que dá suporte no crescimento da produção vegetal/animal na região e no país, gerando alimentos, emprego e renda, além de fortalecer a importância do homem no campo. A extensão universitária faz com que à comunidade acadêmica, viva uma relação transformadora entre Universidade e Sociedade. Além disso, no retorno à Universidade, docentes e discentes trarão um aprendizado que, submetido à reflexão teórica, será acrescido àquele conhecimento (GABRIEL et al., 2015).
O correram melhorias das infraestruturas, ou seja, melhorias nas instalações físicas da UFGD tais como laboratório, equipamento e instalação de irrigação e, consequentemente, manutenção da unidade demonstrativa na Faculdade de Ciência Agrárias visando dar continuidade nos eventos de dia de campo. Ocorreu também uma integração acadêmica com articulação entre o ensino e a pesquisa, onde à estrutura montada serve para aulas práticas na graduação e pós-graduação e de pesquisa por meio do fornecimento de feno para os animais conforme os trabalhos publicados (OLIVEIRA et al., 2013; 2014; 2016; MONÇÃO et al., 2014; 2016). 
Houve integração entre as áreas do conhecimento nos aspectos da interdisciplinaridade e multidisciplinaridade entre os discentes e docentes da Faculdade de Ciências Agrárias-FCA e pela Faculdade de Ciências Exatas e Tecnologia- FACET. A interdisciplinaridade depende fundamentalmente de uma atitude de colaboração dos pesquisadores frente ao desafio de uma prática coletiva, com o objetivo de se produzir conhecimento novo, unitário e crítico.

A divulgação dos resultados permitirá que outras pessoas possam avaliar e, possivelmente, utilizar as informações que foram geradas e também poderá fazer com que a sociedade possa tomar conhecimento dos resultados de um trabalho de pesquisa e o que este representa para a coletividade (BROFMAN, 2012).

O trabalho promoveu a capacitação de recursos humanos para realização da atividade de dia de campo e orientação para os funcionários da fazenda da UFGD, onde se verifica que o treinamento é um processo educacional que pode gerar crescimento e mudanças, melhorando assim o espírito de equipe, integração e criatividade (OLIVEIRA et al., 2012). A capacitação do indivíduo é muito importante, pois será o que irá determinar os principais valores, permitindo analisar as possíveis particularidades de cada pessoa, gerando assim um melhor aproveitamento da atividade.

A difusão e divulgação da tecnologia do dia de Campo gerou um grande impacto. Os resultados obtidos para a comunidade, público alvo, foram efetivos e eficientes por meio do conhecimento adquirido ocorrendo replicação da produção de feno. A produção de feno para uso como fonte de volumoso na dieta do rebanho é uma tecnologia pouco utilizada no Brasil, mas, se entendidas suas práticas de produção, observa-se que é técnica de fácil utilização e pode contribuir de forma significativa para elevar os índices zootécnicos e viabilizar economicamente o empreendimento agropecuário, tanto de forma direta como indireta (EVANGELISTA e LIMA, 2013).

O impacto econômico que ocorreu pela a produção de fardos de feno para alimentação animal da UFGD foi expressivo, o que levou a uma economia no sistema de produção. São vários os fatores que levam a baixa produtividade, porém a estacionalidade na oferta de forragem aos rebanhos, principalmente no período seco do ano (junho a outubro), gera impacto direto em todos os indicadores zootécnicos e reprodutivos nas atividades, como produção de leite, índice de parição, taxa de natalidade, mortalidade, dentre outros. O alimento feno, em relação a 
outros volumosos usualmente utilizados na criação de ruminantes, apresenta muitas vantagens de qualidade e valor nutritivo da forragem, bem como de cunho operacional ou estratégico no dia-a-dia da propriedade (EVANGELISTA e LIMA, 2013).

Houve impacto social devido à relação dos participantes do projeto aliado ao público externo. O impacto social é um sinal importante para o sucesso dos negócios, onde os serviços prestados geram conhecimentos que poderão melhorar a produtividades e contribuir diretamente para aumento de renda das famílias dos participantes. Ao treinar os participantes eles poderão construir infraestrutura física, adquirir matérias primas, transferir tecnologias, e aumentar o acesso a produtos e outros serviços necessários.

O impacto ambiental por meio de adubações orgânicas foi positivo possibilitando uma cobertura de solo adequada colaborando para reconstituir o meio, para o retorno de se seu equilíbrio e para melhoria da qualidade de vida de todos os envolvidos.

\section{Conclusão}

O evento proporcionou uma demonstração teórico-prática dos processos de fenação, com participação de produtores rurais, assentados, quilombolas e discentes de vários cursos. $\mathrm{O}$ evento supriu as necessidades de conhecimento do público, além de oferecer conhecimento de manejo vegetal de forma orgânica.

\section{Agradecimentos}

À Pró-Reitoria de Extensão e Cultura da Universidade Federal da Grande Dourados - UFGD, ao Conselho Nacional de Desenvolvimento Científico e Tecnológico CNPq; ao Núcleo de construção participativa do conhecimento em Agroecologia e Produção Orgânica; ao Centro vocacional tecnológico em Agroecologia e Produção Orgânica, em Mato Grosso do Sul; Fundect e aos órgãos de extensão rural de Mato Grosso do Sul.

\section{Referências}

BARBERO, R.P.; MALHEIROS, E.B.; ARAÚJO, T.L.R.; NAVE, R.L.G.; MULLINIKS, J.T.; BERCHIELLI, T.T.; RUGGIERI, A.C.; REIS, R.A. Combining Marandu grass grazing height andsupplementation level to optimize growth and productivity of yearling bulls, Animal Feed. Science and Technology, v.209, n. 1, p.110-118, 2015.

BROFMN, P.R. Importância das publicações científicas. Cogitare Enfermagem, v.17, n. 3, p. 419-421, 2012. 
DETMANN, E.; SOUZA, M. A.; VALADARES FILHO, S. C.; QUEIROZ, A. C.; BERCHIELLI, T. T.; SALIBA, E. O. S.; CABRAL, L. S.; PINA, D. S.; LADEIRA, M. M.; AZEVEDO, J. A. G. (Ed.). Métodos para análise de alimentos. Visconde do Rio Branco: INCT-Ciência Animal, 2012. $214 \mathrm{p}$.

DIAS, V.V.; SCHULTZ, G.; SCHUSTER, M.S.; TALAMINI, E.; RÉVILLION, J.P. O mercado de alimentos orgânicos: um panorama quantitativo e qualitativo das publicações internacionais. Ambiente \& Sociedade, v. 18, n. 1,p. 161-182, 2015.

EVANGELISTA, A.R.; LIMA, J.A. Produção de feno. Conservação de alimentos para bovinos. Informe Agropecuário, v. 34, n. 277, p. 43-52, 2013.

FEIDEN, A.; ALMEIDA, D. L. de; VITOI, V.; ASSIS, R.L. Processo de conversão de sistemas de produção convencionais para sistemas de produção orgânicos. Cadernos de Ciência e Tecnologia, v. 19,n. 2, p. 179-204, 2002.

GABRIEL, A.M.A.; SOUZA, R.; OLIVEIRA, E.R.; ROSSINI, L.C.; MONÇÃO, F.P.; RAMOS, M.B.M.; GIMENES, L.S.; PEREIRA, T.L.; SILVA, E.C.P. Orientação em apiários noAssentamento Amparo, Dourados-MS. Revista online de Extensão e Cultura Realização, v. 2, n.3, p. 36-41, 2015.

MAZZOLENI, E.M.; NOGUEIRA, J.M. Agricultura orgânica: características básicas do seu produtor. RER, v. 44, n. 02, p. 263-293, 2006.

MONÇÃO, F.P; OLIVEIRA, E.R.;GABRIEL, A.M.A.; SOUZA, R.; MOURA, L.V.;LEMPP, L.; SANTOS, M.V. Degradabilidade ruminal de diferentes gramíneasdo gênero Cynodon spp. em quatro idades de corte. Agrária - Revista Brasileira de Ciências Agrárias, v.9, n.2, p.301-307, 2014.

MONÇÃO, F.P.; OLIVEIRA, E.R.; GABRIEL, A.M.A.; NASCIMENTO, F.A.; PEDROSO, F.W.; FREITAS, L.L. Nutritional parameters of leaf blade from different tropical forages. Scientia Agraria Paranaensis, v. 15, n. 2, p. 185-193, 2016.

OLIVEIRA, E.R.; MONÇÃO, F.P.; GABRIEL, A.M.A.; GÓES, R.H.T.B.; LEMPP, B.; MOURA, L.V. Ruminal degradability of neutral detergent fiber of Cynodon spp. grasses at four regrowth ages. Acta Scientiarum. Animal Sciences, v. 36, n. 2, p. 201-208, 2014 a.

OLIVEIRA, E.R MONÇÃO, F.P.; GORDIN, C.L.; GABRIEL, A.M.A.; LEMPP, B.; SANTOS, M.V.; REIS, S.T.; MOURA, L.V. Ruminal degradability of dry matter of leaves and stem of genotypes of Cynodon spp four ages of regrowth. Semina: Ciências Agrárias, v. 35, n. 5, p. 26592672, 2014 b.

OLIVEIRA, E.R.; MONÇÃO, F.P.; GABRIEL, A.M.A.; SILVA, L.H.X.; CARBONARI, V.M.S.; PEDROSO, F.W.; PEREIRA, T.L.; NASCIMENTO, F.A. Valor nutricional do colmo de gramíneas tropicais. Scientia Agraria Paranaensis, v. 15, n. 3, p. 256-264, 2016 a.

OLIVEIRA, E.R.; GABRIEL, A.M.A.; MONÇÃO, F.P.; FARIAS, M.F.L.; PEIXOTO, P.P.P.; RAMOS, M.B.M.; MOURA, L.V.; PEREIRA, T.L.; GANDRA, J.R.; SANTOS JUNIOR, E.A. Extensão universitária como estratégia de desenvolvimento na Comunidade Quilombola de Dourados/MS. Revista online de Extensão e Cultura Realização, v. 3, n.1, p. 35-44, 2016 b. 
OLIVEIRA, E.R.; MONÇÃO, F.P.;GABRIEL, A.M.A.; FERNANDES, A.R.M.; MOURA, L.V.; NASCIMENTO, F.A. Performance and digestibility in feed lot lambs fed hay based diets. Acta Scientiarum. Animal Sciences, v. 38, 4, p. 425-430, $2016 \mathrm{c}$.

OLIVEIRA, E.R.; MONÇÃO, F.P.; RAMOS, M.B.M.; GABRIEL, A. M. A.; FARIAS, M.F.L.; MOURA, L.V. Práticas extensionistas no desenvolvimento sustentável da comunidade quilombola de Dourados, Mato Grosso do Sul. Em Extensão, v. 11, n. 2, p. 82-95, 2012.

PATTERSON, T.; KLOPFENSTEIN, T. J.; MILTON, T.; BRINK, D. R. Evaluation of the 1996 beef cattle NRC model predictions of intake and gain for calves fed low or medium energy density diets. Nebraska Beef Report, Nebraska, p. 26-29, 2000.MP 73-A.

PEIXOTO, M. Extensão rural no Brasil- uma abordagem histórica da legislação, Texto para Discussão, 48 - ISSN 1983-0645, 2008.

REIS, R. A.; TEIXEIRA, I. A. M. DE A.; SIQUEIRA, G. R. Impacto da qualidade da forragem na produção animal. Anais de Simpósio da $\mathbf{4 3}^{\circ}$ Reunião da SBZ, João Pessoa, PB, 2006.

SCHEIDEMANTEL, S.E.; KLEIN, R.; TEIXEIRA, L.I. A importância da Extensão Universitária: o Projeto Construir. Anais do $2^{\circ}$ Congresso Brasileiro de Extensão Universitária, Belo Horizonte - 12 a 15 de setembro de 2004.

SILVA, A.P.G; SILVA, G.G. Planejando e executando o dia de campo. Recife, instituto agronômico de Pernambuco, IPA, 2013, 38 p. 